\title{
THE CHOW RING OF THE STACK OF RATIONAL CURVES WITH AT MOST 3 NODES
}

\author{
DAMIANO FULGHESU
}

\begin{abstract}
In this paper we explicit the rational Chow ring of the stack $\mathfrak{M}_{0}^{\leq 3}$ consisting of nodal curves of genus 0 with at most 3 nodes: it is a $\mathbb{Q}$-algebra with 10 generators and 11 relations.
\end{abstract}

\section{INTRODUCTION}

In this paper we explicit the ring $A^{*}\left(\mathfrak{M}_{0}^{\leq 3}\right) \otimes \mathbb{Q}$ (Theorem 6.3). Our technique is to compute $A^{*}\left(\mathfrak{M}_{0}^{\leq n}\right) \otimes \mathbb{Q}$ for $n \leq 3$ by induction on $n$. For $n=0$ we have $\mathfrak{M}_{0}^{0}=\operatorname{BP} G L_{2}$, and this case is well understood $[\mathbf{P a n}$.

The inductive step is based on the following fact: if $n \leq 4$, then the top Chern class of the normal bundle of $\mathfrak{M}_{0}^{n}$ into $\mathfrak{M}_{0}^{\leq n}$ is not a 0divisor in $A^{*}\left(\mathfrak{M}_{0}^{n}\right) \otimes \mathbb{Q}$. As a consequence, by an elementary algebraic Lemma (2.1) we can reconstruct the ring $A^{*}\left(\mathfrak{M}_{0}^{\leq n}\right) \otimes \mathbb{Q}$ from the rings $A^{*}\left(\mathfrak{M}_{0}^{\leq n-1}\right) \otimes \mathbb{Q}$ and $A^{*}\left(\mathfrak{M}_{0}^{n}\right) \otimes \mathbb{Q}$, provided that we have an explicit way of extending each class in $A^{*}\left(\mathfrak{M}_{0}^{\leq n-1}\right) \otimes \mathbb{Q}$ to a class in $A^{*}\left(\mathfrak{M}_{0}^{\leq n}\right) \otimes \mathbb{Q}$ (as we have seen in Fulg2 $)$, and then computing the restriction of this extension to $A^{*}\left(\mathfrak{M}_{0}^{n}\right) \otimes \mathbb{Q}$.

We find 10 generators: the classes $\gamma_{\Gamma}$ for all trees $\Gamma$ with at most three nodes (they are 5), plus the Mumford class $k_{2}$. The remaining 4 generators are somewhat new with respect to the tautological classes introduced for stable curves.

The ideal of relations is determined essentially through two useful technical algebraic Lemmas (2.1) and 2.3).

\section{Algebraic lemmas}

Here we state and prove two algebraic lemmas for future reference. The first one is Lemma 4.4 [Ve-Vi]:

Lemma 2.1. Let $A, B$ and $C$ be rings, $f: B \rightarrow A$ and $g: B \rightarrow C$ ring homomorphism. Let us suppose that there exists an homomorphism of abelian groups $\phi: A \rightarrow B$ such that the sequence

$$
A \stackrel{\phi}{\rightarrow} B \stackrel{g}{\rightarrow} C \rightarrow 0
$$


is exact; the composition $f \circ \phi: A \rightarrow A$ is multiplication by a central element $a \in A$ which is not a 0-divisor.

Then $f$ and $g$ induce an isomorphism of ring

$$
(f, g): B \rightarrow A \times_{A /(a)} C,
$$

where the homomorphism $A \stackrel{p}{\rightarrow} A /(a)$ is the projection, while $C \stackrel{q}{\rightarrow}$ $A /(a)$ is induced by the isomorphism $C \simeq B / \operatorname{ker} g$ and the homomorphism of rings $f: B \rightarrow A$.

Proof. Owing to the fact that $a$ is not a 0-divisor we immediately have that $\phi$ is injective. Let us observe that the map $(f, g): B \rightarrow$ $A \times_{A /(a)} C$ is well defined for universal property and for commutation of the diagram:

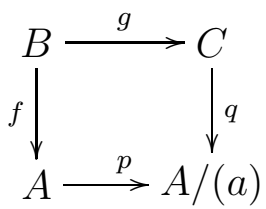

Now let us exhibit the inverse function $A \times_{A /(a)} C \stackrel{\rho}{\rightarrow} B$. Given $(\alpha, \gamma) \in$ $A \times_{A /(a)} C$, let us chose an element $\beta \in B$ such that $g(\beta)=\gamma$. By definition of $q$ we have that $f(\beta)-\alpha$ lives in the ideal $(a)$ and so (it is an hypothesis on $f \circ \phi)$ there exists in $A$ an element $\tilde{\alpha}$ such that:

$$
f(\beta)-\alpha=f(\phi(\tilde{\alpha}))
$$

from which:

$$
f(\beta-\phi(\tilde{\alpha}))=\alpha
$$

we define then $\rho(\alpha, \gamma):=\beta-\phi(\tilde{\alpha})$. In order to verify that it is a good definition, let us suppose that there exist an element $\beta_{0} \in B$ such that $(f, g)\left(\beta_{0}\right)=0$, to be precise there exists an element $\alpha_{0} \in A$ such that: $\phi\left(\alpha_{0}\right)=0$ and furthermore

$$
0=f\left(\phi\left(\alpha_{0}\right)\right)=a \alpha_{0}
$$

but we have that $a$ is not a divisor by zero, so necessary we have $\alpha_{0}=0$ and $\beta_{0}=0$. we conclude by noting that from the definition of $\rho$ we have immediately that it is an isomorphism.

Remark 2.2. The Lemma 2.1 will be used for computing the Chow ring of $\mathfrak{M}_{0}^{\leq k+1}$ when the rings $A^{*}\left[\mathfrak{M}_{0}^{\leq k}\right] \otimes \mathbb{Q}$ and $A^{*}\left[\mathfrak{M}_{0}^{k+1}\right] \otimes \mathbb{Q}$ are known. As a matter of fact, given an Artin stack $\mathcal{X}$ and a closed Artin substack $y \stackrel{i}{\rightarrow} X$ of positive codimension, we have the exact sequence of groups (see Kre Section 4)

$$
A^{*}(y) \otimes \mathbb{Q} \stackrel{i_{*}}{\rightarrow} A^{*}(X) \otimes \mathbb{Q} \stackrel{j^{*}}{\rightarrow} A^{*}(\mathcal{U}) \rightarrow 0
$$


By using the Self-intersection Formula, it follows that:

$$
i^{*} i_{*} 1=i^{*}[y]=c_{t o p}\left(\mathcal{N}_{y / x}\right)
$$

when $c_{t o p}\left(\mathcal{N}_{y / x}\right)$ is not 0 -divisor we can apply the Lemma.

We will also use the following algebraic Lemma:

Lemma 2.3. Given the morphisms

$$
A_{1} \stackrel{p_{1}}{\longrightarrow} \bar{A}_{1} \rightarrow B \leftarrow \bar{A}_{2} \stackrel{p_{2}}{\longleftarrow} A_{2}
$$

in the category of rings, where the maps $p_{1}$ and $p_{2}$ are quotient respectively for ideals $I_{1}$ and $I_{2}$. Then it defines an isomorphism

$$
\bar{A}_{1} \times_{B} \bar{A}_{2} \cong \frac{A_{1} \times_{B} A_{2}}{\left(I_{1}, I_{2}\right)} .
$$

Proof. Let us consider the map

$$
\left(p_{1}, p_{2}\right): A_{1} \times_{B} A_{2} \rightarrow \bar{A}_{1} \times_{B} \bar{A}_{2},
$$

by surjectivity of $p_{1}$ e $p_{2}$ this map is surjective, while the kernel is the ideal $\left(I_{1}, I_{2}\right)$.

\section{THE OPEN SUBSTACK $\mathfrak{M}_{0}^{0}$}

\section{$\Gamma:=\bullet$}

As we have seen in Fulg1

$$
\mathfrak{M}_{0}^{\Gamma} \simeq \operatorname{BAut}(C) .
$$

we have that the stack $\mathfrak{M}_{0}^{0}$ is the classifying space of $\mathbb{P} G l_{2}$. Owing to the fact that $\mathbb{P} G l_{2} \cong S_{3}$ and following [Pan] we have

$$
A^{*}\left(\mathfrak{M}_{0}^{0}\right) \otimes \mathbb{Q} \cong \mathbb{Q}\left[c_{2}\left(\mathfrak{s l}_{2}\right)\right]
$$

Since (as shown in Fulg2 $) c_{2}\left(\mathfrak{s l}_{2}\right)=(1 / 2) k_{2}$, we can write

\section{Proposition 3.1.}

$$
A^{*}\left(\mathfrak{M}_{0}^{0}\right) \otimes \mathbb{Q}=\mathbb{Q}\left[k_{2}\right]
$$




\section{The FIRST STRATUM}

\section{$\Gamma:=\bullet$}

We order the two components. The automorphism group is $\mathfrak{C}_{2} \ltimes(E \times$ $E$ ), (where $\mathfrak{C}_{2}$ is the order two multiplicative group) and the action of its generator $\tau$ over $E \times E$ exchanges the components.

Then the induced action of $\tau$ on the $\operatorname{ring} A_{E \times E}^{*} \otimes \mathbb{Q} \cong \mathbb{Q}\left[t_{1}, t_{2}\right]$ exchanges the first Chern classes $t_{1}$ and $t_{2}$. The invariant polynomials are the symmetric ones which are algebrically generated by: $\left\{\left(t_{1}+\right.\right.$ $\left.\left.t_{2}\right) / 2,\left(t_{1}^{2}+t_{2}^{2}\right) / 2\right\}$. By recalling the description of Mumford classes given in Fulg2, we have $A^{*}\left(\mathfrak{M}_{0}^{1}\right) \otimes \mathbb{Q}=\mathbb{Q}\left[\mathcal{K}_{1}, \mathcal{K}_{2}\right]$. Let us consider the two inclusions $i$ and $j$ (respectively closed and open immersions) and the étale covering $\phi$

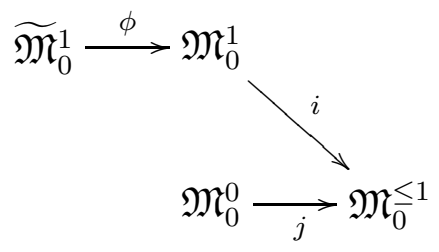

we obtain the following exact sequence

$$
A^{*}\left(\mathfrak{M}_{0}^{1}\right) \otimes \mathbb{Q} \stackrel{i_{*}}{\rightarrow} A^{*}\left(\mathfrak{M}_{0}^{\leq 1}\right) \otimes \mathbb{Q} \stackrel{j^{*}}{\rightarrow} A^{*}\left(\mathfrak{M}_{0}^{0}\right) \otimes \mathbb{Q} \rightarrow 0
$$

for what we have seen we have:

$$
\mathbb{Q}\left[k_{1}, k_{2}\right] \stackrel{i_{*}}{\rightarrow} A^{*}\left(\mathfrak{M}_{0}^{\leq 1}\right) \otimes \mathbb{Q} \stackrel{j^{*}}{\rightarrow} \mathbb{Q}\left[\kappa_{2}\right] \rightarrow 0 .
$$

Now we have that the first Chern class of the normal bundle $N_{\mathfrak{M}_{0}^{1}}\left(\mathfrak{M}_{0}^{\leq 1}\right)$ is

$$
i^{*} i_{*}\left[\mathfrak{M}_{0}^{1}\right]=\phi_{*} \frac{1}{2}\left(t_{1}+t_{2}\right)=-\kappa_{1}
$$

Since $A^{*} \mathfrak{M}_{0}^{1}$ is an integral domain we can apply Lemma (2.1) and obtain the ring isomomorphism

$$
A^{*}\left(\mathfrak{M}_{0}^{\leq 1}\right) \otimes \mathbb{Q} \cong \mathbb{Q}\left[k_{1}, k_{2}\right] \times_{\mathbb{Q}\left[k_{2}\right]} \mathbb{Q}\left[k_{2}\right] \cong \mathbb{Q}\left[k_{1}, k_{2}\right] .
$$

where the map $q: \mathbb{Q}\left[k_{2}\right] \rightarrow \mathbb{Q}\left[k_{1}, k_{2}\right] /\left(k_{1}\right)=\mathbb{Q}\left[k_{2}\right]$ tautologically sends $k_{2}$ into $k_{2}$.

So we have

\section{Proposition 4.1.}

$$
A^{*}\left(\mathfrak{M}_{0}^{\leq 1}\right) \otimes \mathbb{Q} \cong \mathbb{Q}\left[k_{1}, k_{2}\right]
$$




\section{The SECOND STRATUM}

$\Gamma:=\bullet \longrightarrow$ We order the two components with one node.

In this case the group of automorphism of the fiber is

$$
\operatorname{Aut}\left(C^{\Gamma}\right) \cong \mathfrak{C}_{2} \ltimes\left(\mathbb{G}_{\mathbf{m}} \times E \times E\right)=: \mathfrak{C}_{2} \ltimes H,
$$

where the action of $\tau$ sends an element $g \in \mathbb{G}_{\mathbf{m}}$ into $g^{-1}$ and exchange the components isomorphic to $E$.

We can identify $A^{*}\left(\mathrm{~B}\left(\mathbb{G}_{\mathbf{m}}\right)^{3}\right)$ with $A^{*}\left(\widetilde{\mathfrak{M}}_{0}^{\Gamma}\right)$ and $A^{*}\left(\operatorname{BAut}(\Gamma) \ltimes\left(\mathbb{G}_{\mathbf{m}}\right)^{3}\right)$ with $A^{*}\left(\mathfrak{M}_{0}^{\Gamma}\right)$. Set

$$
t_{1}=\psi^{1}(\infty, 1) \quad t_{2}=\psi^{1}(\infty, 2) \quad r=\psi^{2}(\infty)
$$

the action induced by $\tau$ on these classes is $\tau\left(r, t_{1}, t_{2}\right)=\left(-r, t_{2}, t_{1}\right)$. With reference to the map

$$
\mathrm{B}\left(\mathbb{G}_{\mathbf{m}}\right)^{3} \stackrel{\phi}{\rightarrow} \operatorname{BAut}(\Gamma) \ltimes\left(\mathbb{G}_{\mathbf{m}}\right)^{3}
$$

we recall that $\phi^{*}$ is a ring isomorphism between $A^{*}\left(\mathfrak{M}_{0}^{1}\right) \otimes \mathbb{Q}$ and $A^{*}\left(\mathrm{~B}\left(\mathbb{G}_{\mathbf{m}}\right)^{3}\right)^{\mathfrak{C}_{2}}$.

We can describe $A^{*}(\mathrm{~B} H) \otimes \mathbb{Q}=\mathbb{Q}\left[r, t_{1}, t_{2}\right]$ as the polynomial ring in $r$ with coefficients in $\mathbb{Q}\left[t_{1}, t_{2}\right]$, so we write a polynomial $P\left(r, t_{1}, t_{2}\right)$ as $\sum_{i=0}^{k} r^{i} P_{i}\left(t_{1}, t_{2}\right)$.

The polynomial $P$ is invariant for the action of $\tau$ if and only if the coefficients of the powers of $r$ in $P\left(r, t_{1}, t_{2}\right)$ are equal to those of the polynomial $P\left(-r, t_{2}, t_{1}\right)$.

That is to say that $P_{i}$ with even index are invariant for the exchange of $t_{1}$ and $t_{2}$, while those with odd index are anti-invariant. An anti-invariant polynomial $Q$ is such that $Q\left(t_{1}, t_{2}\right)+Q\left(t_{2}, t_{1}\right)=0$ and consequently it is the product of $\left(t_{1}-t_{2}\right)$ by an invariant polynomial. It is furthermore straightforward verifying that any such polynomial is invariant for the action of $\tau$.

So an algebraic system of generators for $\left(A_{\left(\mathbb{G}_{\mathbf{m}}\right)^{3}}^{*} \otimes \mathbb{Q}\right)^{\mathfrak{C}_{2}}$ is given by

$$
u_{1}:=t_{1}+t_{2} \quad u_{2}:=t_{1}^{2}+t_{2}^{2}, \quad u_{3}:=r\left(t_{1}-t_{2}\right), \quad u_{4}:=r^{2} .
$$

We know that

$$
\begin{aligned}
& \phi^{*} k_{1}=-u_{1} \\
& \phi^{*} k_{2}=-u_{2} \\
& \phi^{*}\left(\gamma_{2}\right)=\left(t_{1}-r\right)\left(t_{2}+r\right)=\frac{1}{2}\left(u_{1}^{2}-u_{2}\right)+u_{3}-u_{4}
\end{aligned}
$$

where $\gamma_{2}=c_{2}\left(\mathcal{N}_{\mathfrak{M}_{0}^{2} / \mathfrak{M}_{0}^{\leq 2}}\right)$, and there exists a class $x \in A^{2} \mathfrak{M}_{0}^{2} \otimes \mathbb{Q}$ such that $u_{3}=\pi^{*} x$. 
Claim 5.1. The ideal of relations is generated on $\mathbb{Q}\left[k_{1}, k_{2}, \gamma_{2}, x\right]$ by the polynomial

$$
\left(2 x+\left(2 k_{2}+k_{1}^{2}\right)\right)^{2}-\left(2 k_{2}+k_{1}^{2}\right)\left(4 \gamma_{2}-k_{1}^{2}\right)=0 .
$$

Proof. From direct computation we have that relation (3) holds and the polynomial is irreducible. On the other hand let us consider the map $f: \mathbb{A}_{\mathbb{Q}}^{3} \rightarrow \mathbb{A}_{\mathbb{Q}}^{4}$ defined as $\left(t_{1}, t_{2}, r\right) \mapsto\left(u_{1}, u_{2}, u_{3}, u_{4}\right)$. If the generic fiber of $f$ is finite then $f\left(\mathbb{A}_{\mathbb{Q}}^{3}\right)$ is an hypersurface in $\mathbb{A}_{\mathbb{Q}}^{4}$ and we have done. Now for semicontinuity it is sufficient to show that a fiber is finite. Let us consider the fiber on 0 . We have that $u_{1}, u_{2}, u_{3}, u_{4}$ are simultaneously zero iff $t_{1}=t_{2}=r=0$.

NOTE: In the following we do not explicit the argument above.

Now set $\eta:=2 x+\left(2 k_{2}+k_{1}^{2}\right)$, we have that $A^{*}\left(\mathfrak{M}_{0}^{2}\right) \otimes \mathbb{Q}$ is isomorphic to the graded ring $\mathbb{Q}\left[k_{1}, k_{2}, \gamma_{2}, \eta\right] / I$ where the ideal $I$ is generated by the polynomial $\eta^{2}-\left(2 k_{2}+k_{1}^{2}\right)\left(4 \gamma_{2}-k_{1}^{2}\right)$. Since $\phi^{*} \phi_{*}$ is multiplication by two, we also have the following relation

$$
\eta=\phi_{*}\left(\frac{1}{2}\left(t_{1}-t_{2}\right)\left(2 r-t_{1}+t_{2}\right)\right) .
$$

Let us consider the cartesian diagram

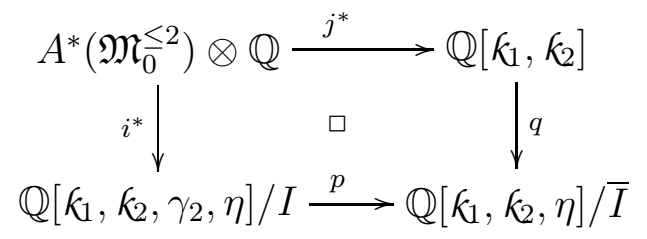

where $\bar{I}$ is the ideal generated by $\eta^{2}+\left(2 k_{2}+\kappa_{1}^{2}\right) k_{1}^{2}$.

The map $q$ is injective so $i^{*}$ is injective too.

We set in $A^{*}\left(\mathfrak{M}_{0}^{\leq 2}\right) \otimes \mathbb{Q}$ the classes $\gamma_{2}:=i_{*} 1$ and $q:=i_{*} \eta$, the ring we want (from injectivity of $i^{*}$ ) is isomorphic to the subring of $A^{*}\left(\mathfrak{M}_{0}^{2}\right) \otimes \mathbb{Q}$ generated by $k_{1}, k_{2}, \gamma_{2}, \gamma_{2} \eta$ so we have

\section{Proposition 5.2.}

$$
A^{*}\left(\mathfrak{M}_{0}^{\leq 2}\right) \otimes \mathbb{Q}=\frac{\mathbb{Q}\left[k_{1}, k_{2}, \gamma_{2}, q\right]}{\left(q^{2}+\gamma_{2}^{2}\left(2 k_{2}+k_{1}^{2}\right)\left(k_{1}^{2}-4 \gamma_{2}\right)\right)}
$$




\section{THE THIRD STRATUM}

The third stratum splits into two components.

\section{The first component}

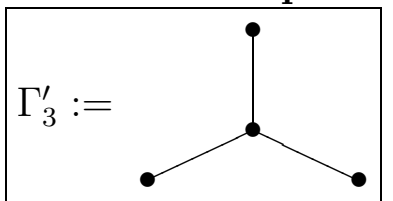

We order components in $\Delta_{1}$. Let us note that the component corresponding to the central vertex has three points fixed by the other three components, consequently, given a permutation of the external vertices, there is an unique automorphism related to the central vertex.

The group $\operatorname{Aut}\left(C^{\Gamma_{3}^{\prime}}\right)$ is therefore isomorphic to $S_{3} \ltimes\left(E^{3}\right)$. We have

$$
A_{E^{3}}^{*} \otimes \mathbb{Q} \cong \mathbb{Q}\left[w_{1}, w_{2}, w_{3}\right],
$$

on which $S_{3}$ acts by permuting the three classes

$$
w_{1}:=\psi_{\infty, 1}^{1} \quad w_{2}:=\psi_{\infty, 2}^{1} \quad w_{3}:=\psi_{\infty, 3}^{1}
$$

So we have

$$
\begin{aligned}
& \phi^{*} k_{1}=-\left(w_{1}+w_{2}+w_{3}\right), \\
& \phi^{*} k_{2}:=-\left(w_{1}^{2}+w_{2}^{2}+w_{3}^{2}\right), \\
& \phi^{*} k_{3}:=-\left(w_{1}^{3}+w_{2}^{3}+w_{3}^{3}\right)
\end{aligned}
$$

conesequently

$$
A^{*}\left(\mathfrak{M}_{0}^{\Gamma_{3}^{\prime}}\right) \otimes \mathbb{Q} \cong \mathbb{Q}\left[k_{1}, k_{2}, k_{3}\right] .
$$

We fix the following notation

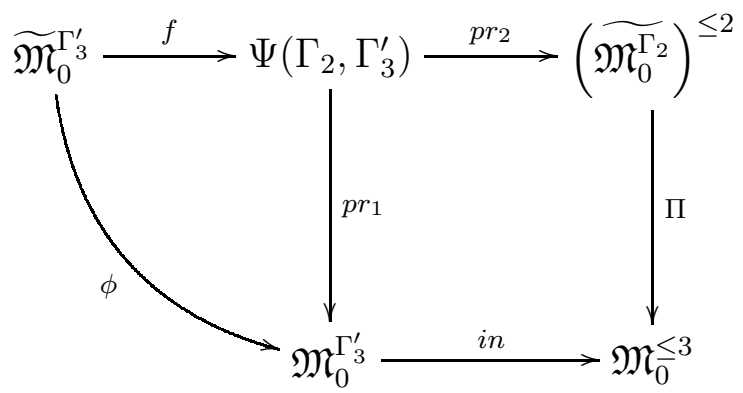

where $f$ is the union of all $f_{\alpha}$.

First of all let us notice that the class $\phi^{*} \gamma_{3}^{\prime}:=\pi^{*} c_{3}\left(\mathcal{N}_{i}\right)=w_{1} w_{2} w_{3}$ depends on Mumford classes in the following way $6 \gamma_{3}^{\prime}=-\left(k_{1}^{3}-3 k_{1} k_{2}+\right.$ $\left.2 k_{3}\right)$ so we can write

$$
A^{*}\left(\mathfrak{M}_{0}^{\Gamma_{3}^{\prime}}\right) \otimes \mathbb{Q}=\mathbb{Q}\left[\kappa_{1}, \kappa_{2}, \gamma_{3}^{\prime}\right]
$$


The restriction of $\gamma_{2}$ to $A^{*}\left(\mathfrak{M}_{0}^{\Gamma_{3}^{\prime}}\right)$ is

$$
\begin{aligned}
\gamma_{2}:=p r_{1 *} \frac{1}{2} c_{2}\left(p r_{1}^{*}\left(\mathcal{N}_{i n}\right) / \mathcal{N}_{p r_{2}}\right) & =\phi_{*} f^{*} \frac{1}{2} c_{2}\left(p r_{1}^{*}\left(\mathcal{N}_{i n}\right) / \mathcal{N}_{p r_{2}}\right) \\
& =\frac{1}{2} \pi_{*}\left(w_{1} w_{3}\right)
\end{aligned}
$$

from which $\phi^{*} \gamma_{2}=w_{1} w_{3}+w_{1} w_{2}+w_{2} w_{3}$ consequently, by writing $2 \gamma_{2}=$ $k_{1}^{2}-k_{2}$ we have

$$
A^{*}\left(\mathfrak{M}_{0}^{\Gamma_{3}^{\prime}}\right) \otimes \mathbb{Q}=\mathbb{Q}\left[\kappa_{1}, \gamma_{2}, \gamma_{3}^{\prime}\right] .
$$

In order to restrict the class $q$ let us notice that we can write

$$
f^{*} r=0 \quad f^{*} t_{1}=w_{1} \quad f^{*} t_{2}=w_{3}
$$

from which we have

$$
f^{*}\left(\frac{1}{2}\left(t_{1}-t_{2}\right)\left(2 t-t_{1}+t_{2}\right)\right)=-\frac{1}{2}\left(w_{1}-w_{3}\right)^{2}
$$

and so

$$
\begin{aligned}
\phi^{*} q & =\phi^{*} \phi_{*}\left(-\frac{1}{2}\left(w_{1}-w_{3}\right)^{2} w_{1} w_{3}\right) \\
& =-\left(\left(w_{1}-w_{3}\right)^{2} w_{1} w_{3}+\left(w_{1}-w_{2}\right)^{2} w_{1} w_{2}+\left(w_{2}-w_{3}\right)^{2} w_{2} w_{3}\right)
\end{aligned}
$$

we can therefore write $q=-\gamma_{2}\left(k_{1}^{2}-4 \gamma_{2}\right)+3 \gamma_{3}^{\prime} k_{1}$. With reference to the inclusions

$$
\mathfrak{M}_{0}^{\Gamma_{3}^{\prime}} \stackrel{i}{\rightarrow} \mathfrak{M}_{0}^{\leq 2} \cup \mathfrak{M}_{0}^{\Gamma_{3}^{\prime}} \stackrel{j}{\leftarrow} \mathfrak{M}_{0}^{\leq 2}
$$

we have the fiber square:

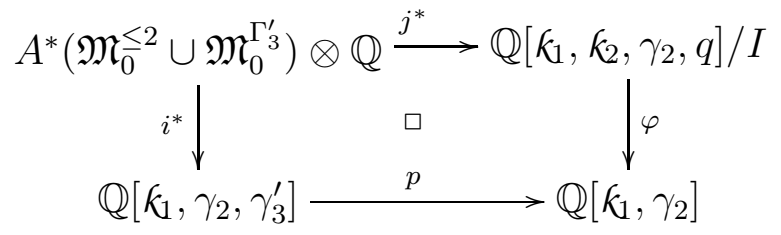

where $I$ is the ideal generated by the polynomial $q^{2}-\gamma_{2}^{2}\left(2 k_{2}-k_{1}^{2}\right)\left(k_{1}^{2}-\right.$ $4 \gamma_{2}$ ) and the map $\varphi$ is surjective and such that

$$
\operatorname{ker} \varphi=\left(q+\gamma_{2}\left(k_{1}^{2}-4 \gamma_{2}\right), \quad k_{2}+2 \gamma_{2}-k_{1}^{2}\right) .
$$

Now let us observe that from Lemma (2.3) the ring in question is isomorphic to

$$
A /(0, I):=\frac{\mathbb{Q}\left[k_{1}, \gamma_{2}, \gamma_{3}^{\prime}\right] \times_{\mathbb{Q}\left[k_{1}, \gamma_{2}\right]} \mathbb{Q}\left[k_{1}, k_{2}, \gamma_{2}, q\right]}{(0, I)} .
$$

Set, with abuse of notation

$$
\begin{array}{rcc}
k_{1}:=\left(k_{1}, k_{1}\right) & \gamma_{2}:=\left(\gamma_{2}, \gamma_{2}\right) & \gamma_{3}^{\prime}:=\left(\gamma_{3}^{\prime}, 0\right) \\
k_{2}:=\left(k_{1}^{2}-2 \gamma_{2}, k_{2}\right) & q:=\left(-\gamma_{2}\left(k_{1}^{2}-4 \gamma_{2}\right), q\right)
\end{array}
$$


Straightforward arguments lead us to state the following

Lemma 6.1. The classes $k_{1}, \gamma_{2}, \gamma_{3}^{\prime}, k_{2}, q$ generate the ring $A$.

Let us compute the ideal of relations. Let $T\left(\kappa_{1}, \gamma_{2}, \gamma_{3}^{\prime}, k_{2}, q\right)$ be a polynomial in $\mathbb{Q}\left[k_{1}, \gamma_{2}, \gamma_{3}^{\prime}, k_{2}, q\right]$. It is zero in $A$ iff

$$
\begin{aligned}
& T\left(\kappa_{1}, \gamma_{2}, \gamma_{3}^{\prime}, \kappa_{1}^{2}-2 \gamma_{2},-\gamma_{2}\left(\kappa_{1}^{2}-4 \gamma_{2}\right)\right)=0 \text { in } \mathbb{Q}\left[k_{1}, \gamma_{2}, \gamma_{3}^{\prime}\right] \\
& T\left(\kappa_{1}, \gamma_{2}, 0, \kappa_{2}, q\right)=0 \text { in } \mathbb{Q}\left[k_{1}, \gamma_{2}, k_{2}, q\right]
\end{aligned}
$$

in particular this implies that $T$ is in the ideal of $\gamma_{3}^{\prime}$. Consequently the polynomial $T=: \gamma_{3}^{\prime} \widehat{T}$ is zero in $A$ iff $\widehat{T}\left(\kappa_{1}, \gamma_{2}, \gamma_{3}^{\prime}, k_{1}^{2}-2 \gamma_{2},-\gamma_{2}\left(k_{1}^{2}-\right.\right.$ $\left.\left.4 \gamma_{2}\right)\right)$ is zero in $\mathbb{Q}\left[k_{1}, \gamma_{2}, \gamma_{3}^{\prime}\right]$. The ideal of relations in $A$ is so generated by $\gamma_{3}^{\prime}\left(-k_{2}+2 \gamma_{2}-\kappa_{1}^{2}\right)$ and $\gamma_{3}^{\prime}\left(q+\gamma_{2}\left(\kappa_{1}^{2}-4 \gamma_{2}\right)\right)$.

Finally let us notice that the ideal $(0, I)$ is generated in $A$ by the polynomial $q^{2}+\gamma_{2}^{2}\left(2 k_{2}+k_{1}^{2}\right)\left(\kappa_{1}^{2}-4 \gamma_{2}\right)$. So we can conclude that

$$
A^{*}\left(\mathfrak{M}_{0}^{\leq 2} \cup \mathfrak{M}_{0}^{\Gamma_{3}^{\prime}}\right) \otimes \mathbb{Q}=\mathbb{Q}\left[\kappa_{1}, \kappa_{2}, \gamma_{2}, \gamma_{3}^{\prime}, q\right] / J,
$$

where $J$ is the ideal generated by the polynomials

$$
\begin{aligned}
& q^{2}+\gamma_{2}^{2}\left(2 k_{2}+k_{1}^{2}\right)\left(k_{1}^{2}-4 \gamma_{2}\right) \\
& \gamma_{3}^{\prime}\left(-k_{2}+2 \gamma_{2}-k_{1}^{2}\right) \\
& \gamma_{3}^{\prime}\left(q+\gamma_{2}\left(k_{1}^{2}-4 \gamma_{2}\right)\right)
\end{aligned}
$$

\section{The second component}

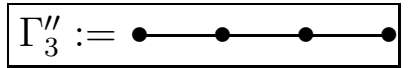

The group of $\operatorname{Aut}\left(C^{\Gamma_{3}^{\prime}}\right)$ is $\mathfrak{C}_{2} \ltimes\left(E \times E \times \mathbb{G}_{\mathbf{m}} \times \mathbb{G}_{\mathbf{m}}\right)$. The action of $\tau$ on this group exchange simultaneously the components related to $\mathbb{G}_{\mathbf{m}}$ and those related to $E$.

We have the isomorphism

$$
A_{E \times E \times \mathbb{G}_{\mathbf{m}} \times \mathbb{G}_{\mathbf{m}}}^{*} \otimes \mathbb{Q} \cong \mathbb{Q}\left[v_{1}, \ldots, v_{4}\right]
$$

where

$$
v_{1}=\psi_{\infty, 1}^{1} \quad v_{2}=\psi_{\infty, 2}^{1} \quad v_{3}=\psi_{\infty, 1}^{2} \quad v_{4}=\psi_{\infty, 2}^{2}
$$

by gluing curves such that the two central components corresponds in the point at infinity.

It follows that the action induced by $\tau$ is

$$
\tau\left(v_{1}, v_{2}, v_{3}, v_{4}\right)=\left(v_{2}, v_{1}, v_{4}, v_{3}\right)
$$

Since $\mathfrak{C}_{2}$ has order 2 , the invariant polynomials are algebraically generated by the invariant polynomials of degree at most two (see. Theorem 7.5 CLO $)$. It is easy to see that a basis for the linear ones is given 
by $u_{1}:=v_{1}+v_{2}, u_{2}:=v_{3}+v_{4}$. For the vector subspace of invariant polynomials of degree two, we can compute a linear basis by using Reynolds' operator

$$
\begin{array}{ll}
u_{3}:=v_{1}^{2}+v_{2}^{2}, & u_{6}:=v_{1} v_{3}+v_{2} v_{4}, \\
u_{4}:=v_{3}^{2}+v_{4}^{2}, & u_{7}:=v_{1} v_{2}, \\
u_{5}:=v_{1} v_{4}+v_{2} v_{3}, & u_{8}:=v_{3} v_{4}
\end{array}
$$

now we note that

$$
u_{6}=u_{1} u_{2}-u_{5}, \quad u_{7}=\left(u_{1}^{2}-u_{3}\right) / 2, \quad u_{8}=\left(u_{2}^{2}-u_{4}\right) / 2
$$

Consequently we can write

$$
A^{*} \mathfrak{M}_{0}^{\Gamma_{3}^{\prime}} \otimes \mathbb{Q} \cong \mathbb{Q}\left[u_{1}, \ldots, u_{5}\right] / I
$$

where $I$ is the ideal generated by the polynomial

$$
2 u_{3} u_{4}+2 u_{1} u_{2} u_{5}-u_{2}^{2} u_{3}-u_{1}^{2} u_{4}-2 u_{5}^{2}
$$

With reference to the degree two covering $\widetilde{\mathfrak{M}}_{0}^{\Gamma_{3}^{\prime}} \stackrel{\phi}{\rightarrow} \mathfrak{M}_{0}^{\Gamma_{3}^{\prime}}$ we have: $-u_{1}=$ $\phi^{*} k_{1}-u_{3}=\phi^{*} k_{2}$. In order to compute the restriction of the closure of the classes $\gamma_{2}$ and $q$ of $\mathfrak{M}_{0}^{\leq 2}$ let us fix the notation of the following diagram

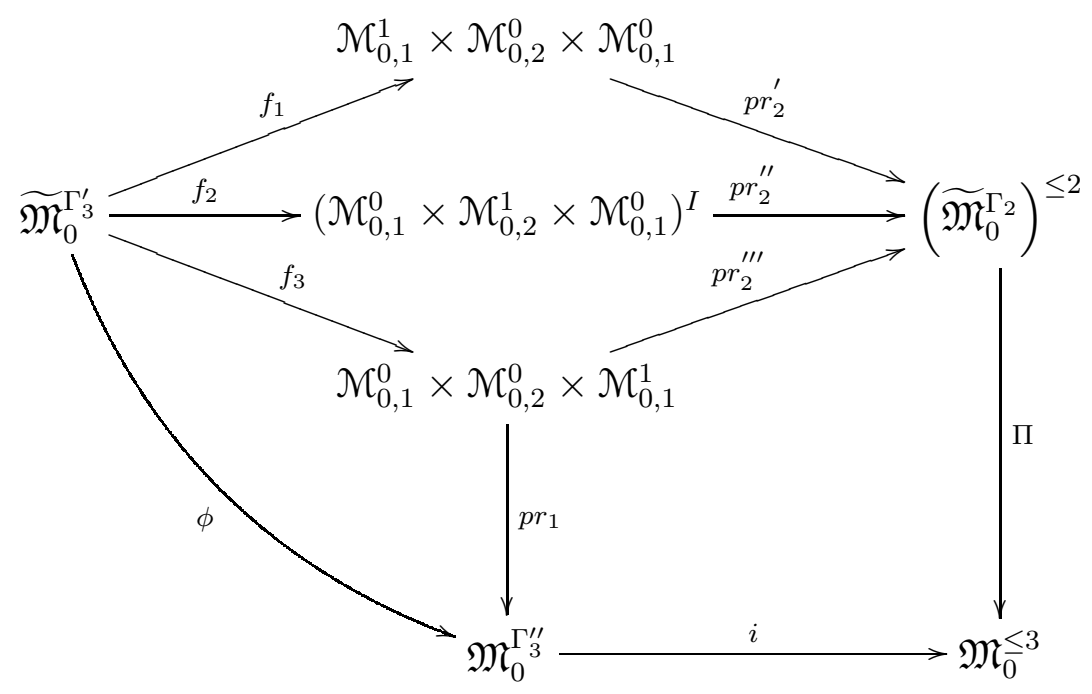

where $\left(\mathcal{M}_{0,1}^{0} \times \mathcal{M}_{0,2}^{1} \times \mathcal{M}_{0,1}^{0}\right)^{I}$ is the component where the marked points of the central curve (which is singular) are on different components.

As $\phi^{*}$ is an isomorphism to the algebra of polynomials which are invariants for the action of $\mathfrak{C}_{2}$, let us choose in $A^{*}\left(\mathfrak{M}_{0}^{\Gamma_{3}^{\prime \prime}}\right) \otimes \mathbb{Q}$ classes $\rho, \lambda, \mu$ such that $u_{2}=\phi^{*} \rho, u_{4}=\phi^{*} \lambda, u_{5}=\phi^{*} \mu$.

First of all let us compute the restriction of the closure of $\gamma_{2} \in$ $A^{*}\left(\mathfrak{M}_{0}^{\leq 2}\right) \otimes \mathbb{Q}$, the polynomial in the classes $\psi$ is $\frac{1}{2}$, we have $c_{3}\left(\phi^{*}\left(\mathcal{N}_{i}\right)\right)=$ 
$\left(v_{1}-v_{3}\right)\left(v_{3}+v_{4}\right)\left(v_{2}-v_{4}\right), c_{1}\left(f_{1}^{*}\left(\mathcal{N}_{p r_{2}^{\prime}}\right)\right)=\left(v_{1}-v_{3}\right), c_{1}\left(f_{2}^{*}\left(\mathcal{N}_{p r_{2}^{\prime \prime}}\right)\right)=\left(v_{3}+\right.$ $\left.v_{4}\right)$ and $c_{1}\left(f_{3}^{*}\left(\mathcal{N}_{p r_{2}^{\prime \prime \prime}}\right)\right)=\left(v_{2}-v_{4}\right)$ from which we obtain the following relations

$$
\begin{aligned}
\phi^{*} \gamma_{3}^{\prime} & =\left(v_{1}-v_{3}\right)\left(v_{3}+v_{4}\right)\left(v_{2}-v_{4}\right) \\
& =\phi^{*}\left(\rho\left(\frac{1}{2}\left(k_{1}^{2}+\rho^{2}-k_{2}-\lambda\right)-\mu\right)\right) \\
\phi^{*} \gamma_{2} & =\left(\left(v_{3}+v_{4}\right)\left(v_{2}-v_{4}\right)+\left(v_{1}-v_{3}\right)\left(v_{2}-v_{4}\right)+\left(v_{1}-v_{3}\right)\left(v_{3}+v_{4}\right)\right) \\
& =-\rho k_{1}-\mu+\frac{1}{2}\left(k_{1}^{2}-\rho^{2}+k_{2}-\lambda\right)
\end{aligned}
$$

In order to have $\gamma_{2}$ among the generators, set

$$
\lambda=-2 \rho k_{1}-2 \mu+k_{1}^{2}-\rho^{2}+k_{2}-2 \gamma_{2}
$$

the equation (4) becomes

$$
K: \sigma^{2}-\left(2 k_{2}+k_{1}^{2}\right)\left(\left(-k_{1}+3 \rho\right)\left(\kappa_{1}+\rho\right)-4 \gamma_{2}\right)
$$

where we have set $\sigma=2 \mu-2 k_{2}-k_{1}^{2}+k_{1} \rho$. Then we can write the $\operatorname{ring} A^{*}\left(\mathfrak{M}_{0}^{\Gamma_{3}^{\prime \prime}}\right) \otimes \mathbb{Q}$ as $\mathbb{Q}\left[k_{1}, \rho, k_{2}, \gamma_{2}, \sigma\right] / K$. In the new basis we have $\gamma_{3}^{\prime}=\rho\left(\rho^{2}-\rho k_{1}+\gamma_{2}\right)$. Let us restricts the closure of $q \in A^{*}\left(\mathfrak{M}_{0}^{\leq 2}\right) \otimes \mathbb{Q}$ to $\mathfrak{M}_{0}^{\Gamma_{1}}$. We recall that the related polynomial in classes $\psi$ of $\widetilde{M}_{0}^{\Gamma_{2}}$ is $\frac{1}{2}\left(t_{1}-t_{2}\right)\left(2 r-t_{1}+t_{2}\right)$. On $\mathcal{M}_{0,1}^{1} \times \mathcal{M}_{0,2}^{0} \times \mathcal{M}_{0,1}^{0}$ we have $f_{1}^{*} t_{1}=v_{3} \quad f_{1}^{*} t_{2}=$ $v_{2} \quad f_{1}^{*} r=-v_{4}$, on $\left(\mathcal{M}_{0,1}^{0} \times \mathcal{M}_{0,2}^{1} \times \mathcal{M}_{0,1}^{0}\right)^{I}$ we have $f_{2}^{*} t_{1}=v_{1} \quad f_{2}^{*} t_{2}=$ $v_{2} \quad f_{2}^{*} r=\frac{\psi_{\infty}^{2}-\psi_{0}^{2}}{2}=\frac{v_{3}-v_{4}}{2}$, and in the end on $\mathcal{M}_{0,1}^{0} \times \mathcal{M}_{0,2}^{0} \times \mathcal{M}_{0,1}^{1}$ we have $f_{3}^{*} t_{1}=v_{1} \quad f_{3}^{*} t_{2}=v_{4} \quad f_{3}^{*} r=v_{3}$, consequently the polynomials related to the three components of $\Psi\left(\Gamma_{2}, \Gamma_{3}^{\prime \prime}\right)$ are $P_{1}=\frac{1}{2}\left(v_{3}-v_{2}\right)\left(v_{2}-v_{3}-2 v_{4}\right)$, $P_{2}=\frac{1}{2}\left(v_{1}-v_{2}\right)\left(v_{4}-v_{3}-v_{1}+v_{2}\right)$ and $P_{3}=\frac{1}{2}\left(v_{4}-v_{1}\right)\left(v_{1}-2 v_{3}-v_{4}\right)$ from which

$$
\phi^{*} q=2 \sum_{\alpha=1}^{3}\left(P_{\alpha} N_{\alpha}\right)=\phi^{*}\left(\left(3 \rho+\kappa_{1}\right) \gamma_{3}^{\prime \prime}+\left(\rho^{2}-\gamma_{2}\right) \sigma\right)
$$

this means that the image of $q$ in $B:=A^{*}\left(\mathfrak{M}_{0}^{\Gamma_{3}^{\prime \prime}}\right) \otimes \mathbb{Q} / \gamma_{3}^{\prime \prime}$ is $\left(\rho^{2}-\gamma_{2}\right) \sigma$. Further let us notice that the image of $\gamma_{3}^{\prime}$ in $B$ is 0 .

In order to compute $A^{*}\left(\mathfrak{M}_{0}^{\leq 3}\right) \otimes \mathbb{Q}$, let us consider its isomorphism with the fibered product

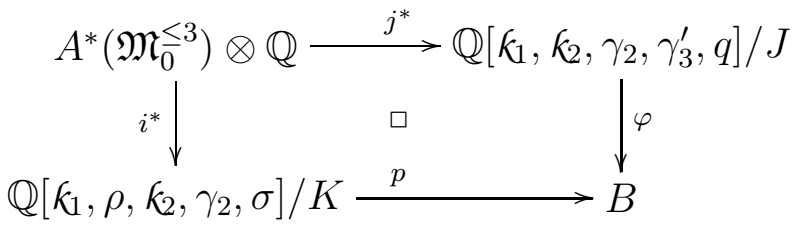


With reference to the fiber square

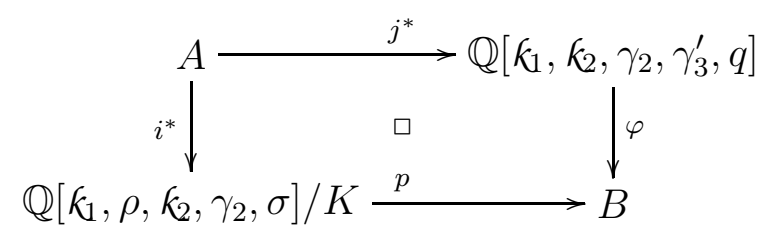

we have that the ring in question is isomorphic to the quotient $A /(0, J)$.

Now we look for generators of the ring $A$.

Again a straightforward argument leads us to state

Lemma 6.2. The following elements of $A$

$$
\begin{array}{lll}
k_{1}:=\left(k_{1}, k_{1}\right) ; & k_{2}:=\left(k_{2}, k_{2}\right) ; & \gamma_{2}:=\left(\gamma_{2}\right) \\
\gamma_{3}^{\prime}:=\left(0, \gamma_{3}^{\prime}\right) ; & q:=\left(\left(\rho^{2}-\gamma_{2}\right) \sigma, q\right) ; & \gamma_{3}^{\prime \prime}:=\left(\gamma_{3}^{\prime \prime}, 0\right) \\
r:=\left(\gamma_{3}^{\prime \prime} \rho, 0\right) ; & s:=\left(\gamma_{3}^{\prime \prime} \sigma, 0\right) & t:=\left(\gamma_{3}^{\prime \prime} \rho^{2}, 0\right) \\
& u:=\left(\gamma_{3}^{\prime \prime} \rho \sigma, 0\right) &
\end{array}
$$

are generators of the ring.

Now let us call $\widetilde{A}$ the $\operatorname{ring} \mathbb{Q}\left[\kappa_{1}, \kappa_{2}, \gamma_{2}, \gamma_{3}^{\prime}, \gamma_{3}^{\prime \prime}, q, r, s, t, u\right]$. We have by fact defined a surjective homomorphism $a: \widetilde{A} \rightarrow A$; we call again $j^{*}$ and $i^{*}$ their composition with $a$, we have $\operatorname{ker} a=\operatorname{ker} i^{*} \cap \operatorname{ker} j^{*}$. Now, for what we've seen, we have:

$$
\begin{aligned}
\operatorname{ker} i^{*} & =\left(\begin{array}{l}
\gamma_{3}^{\prime} \\
r^{2}-\gamma_{3}^{\prime \prime} t \\
r s-\gamma_{3}^{\prime \prime} u \\
s^{2}-\left(\gamma_{3}^{\prime \prime}\right)^{2}\left(2 k_{2}+k_{1}^{2}\right)\left(\left(-k_{1}+2 \rho\right)\left(k_{1}+\rho\right)+4 \gamma_{2}\right)
\end{array}\right) \\
\operatorname{ker} j^{*} & =\left(\gamma_{3}^{\prime \prime}, r, s, t, u\right)
\end{aligned}
$$

and so:

$$
\operatorname{ker} a=\left(\begin{array}{l}
\gamma_{3}^{\prime} \gamma_{3}^{\prime \prime} ; \quad \gamma_{3}^{\prime} r ; \quad \gamma_{3}^{\prime} s ; \quad \gamma_{3}^{\prime} t ; \quad \gamma_{3}^{\prime} u ; \\
r^{2}-\gamma_{3}^{\prime \prime} t ; \quad r s-\gamma_{3}^{\prime \prime} u ; \\
s^{2}-\left(\gamma_{3}^{\prime \prime}\right)^{2}\left(2 k_{2}+\kappa_{1}^{2}\right)\left(\left(-k_{1}+2 \rho\right)\left(k_{1}+\rho\right)+4 \gamma_{2}\right)
\end{array}\right)
$$

We make the quotient of $A=A^{\prime} /$ ker $a$ by the ideal $(0, J)$ and we obtain the following:

Theorem 6.3. The ring $A^{*}\left(\mathfrak{M}_{0}^{\leq 3}\right) \otimes \mathbb{Q}$ is:

$$
\mathbb{Q}\left[\kappa_{1}, k_{2}, \gamma_{2}, \gamma_{3}^{\prime}, \gamma_{3}^{\prime \prime}, q, r, s, t, u\right] / L
$$


where $L$ is the ideal generated by the polynomials

$$
\begin{aligned}
& \gamma_{3}^{\prime}\left(-k_{2}+2 \gamma_{2}-k_{1}^{2}\right) ; \quad \gamma_{3}^{\prime}\left(q+\gamma_{2}\left(k_{1}^{2}-4 \gamma_{2}\right)\right) ; \\
& \gamma_{3}^{\prime} \gamma_{3}^{\prime \prime} ; \gamma_{3}^{\prime} r ; \quad \gamma_{3}^{\prime} s ; \gamma_{3}^{\prime} t ; \quad \gamma_{3}^{\prime} u ; \\
& r^{2}-\gamma_{3}^{\prime \prime} t ; \quad r s-\gamma_{3}^{\prime \prime} u ; \\
& q^{2}+\left(\gamma_{2}\right)^{2}\left(2 k_{2}+k_{1}^{2}\right)\left(k_{1}^{2}-4 \gamma_{2}\right) ; \\
& s^{2}-\left(2 k_{2}+k_{1}^{2}\right)\left(\left(-k_{1} \gamma_{3}^{\prime \prime}+2 r\right)\left(k_{1} \gamma_{3}^{\prime \prime}+r\right)+4 \gamma_{2}\left(\gamma_{3}^{\prime \prime}\right)^{2}\right) \\
& \text { REFERENCES }
\end{aligned}
$$

\section{REFERENCES}

[CLO] D.Cox, J.Little, D.O'Shea: Ideals, Varieties, and Algorithms 2nd ed, Undergraduate Text in Math, Springer-Verlag New York, 1998.

[Fulg1] D. Fulghesu, The Stack of Rational Nodal Curves, preprint.

[Fulg2] D. Fulghesu, Tautological Classes of the Stack of Rational Nodal Curves, preprint.

[Kre] A. Kresch: Cycle groups for Artin stacks; Invent. Math. 138 (1999) 495-536.

[Pan] R. Pandharipande: Equivariant Chow rings of $O(k), S O(2 k+1)$ and $S O(4)$; J. Reine Angew. Math. 496 (1998) 131-148.

[Ve-Vi] G. Vezzosi, A. Vistoli: Higher algebraic $K$-theory for actions of diagonalizable groups, Invent. Math. 153 (2003) 1-44.

Department of Mathematics, University of Missouri, Columbia, MO 65211

E-mail address: damiano@math.missouri.edu 